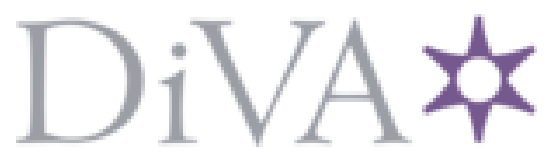

http://www.diva-portal.org

This is the published version of a paper presented at Conference on Lasers and ElectroOptics (CLEO) 2019.

Citation for the original published paper:

Ottonello-Briano, F., Errando-Herranz, C., Rödjegård, H., Martin, H., Sohlström, H. et al. (2019)

Carbon Dioxide Sensing with Low-confinementHigh-sensitivity Mid-IR

SiliconWaveguides

In: Conference on Lasers and Electro-Optics 2019: CLEO: Science and Innovations, STh1F.3

https://doi.org/10.1364/CLEO_SI.2019.STh1F.3

N.B. When citing this work, cite the original published paper.

Permanent link to this version:

http://urn.kb.se/resolve?urn=urn:nbn:se:kth:diva-253841 


\title{
Carbon Dioxide Sensing with Low-confinement High-sensitivity Mid-IR Silicon Waveguides
}

\author{
Floria Ottonello-Briano ${ }^{1}$, Carlos Errando-Herranz ${ }^{1}$, Henrik Rödjegård ${ }^{2}$, \\ Hans Martin ${ }^{2}$, Hans Sohlström ${ }^{1}$, and Kristinn B. Gylfason ${ }^{1}$ \\ ${ }^{1}$ Micro and Nanosystems, KTH Royal Institute of Technology, Malvinas väg 10, 10044 Stockholm, Sweden \\ ${ }^{2}$ Senseair AB, Stationsgatan 12, 82471 Delsbo, Sweden \\ floria@kth.se
}

\begin{abstract}
We present a low-confinement Si waveguide for $4.26 \mu \mathrm{m}$ wavelength and apply it to sense $\mathrm{CO}_{2}$ concentrations down to $0.1 \%$. We demonstrate the highest reported waveguide sensitivity to $\mathrm{CO}_{2}: 44 \%$ of the free-space sensitivity. (c) 2019 The Author(s) OCIS codes: (280.4788) Optical sensing and sensors, (130.3060) Infrared, (230.7370) Waveguides
\end{abstract}

Carbon dioxide $\left(\mathrm{CO}_{2}\right)$ strongly absorbs light at specific wavelengths around $4.26 \mu \mathrm{m}$, i.e. in the mid-infrared (mid-IR) spectral range. This set of absorption peaks does not overlap with those of other gases commonly present in air, such as water vapor (Fig. 1a). This can be exploited to optically sense $\mathrm{CO}_{2}$ selectively and accurately. Furthermore, optical $\mathrm{CO}_{2}$ sensors offer a fast response and minimal drift, without consuming the measured gas [1], but their widespread adoption has so far been limited by their large size and high cost. For this reason, the miniaturization and cost reduction of optical $\mathrm{CO}_{2}$ sensors have gained relevance in recent years.

Miniaturization of optical $\mathrm{CO}_{2}$ sensor to the level required by mobile applications cannot be achieved with the free-space configuration that traditional non-dispersive infrared (NDIR) $\mathrm{CO}_{2}$ sensors use, but it is possible with on-chip photonic waveguides. Such waveguides must support mid-IR light modes with a low confinement, i.e. a large portion of the optical power outside the waveguide core, so that the propagating light can interact with the gas. Previous works reporting $\mathrm{CO}_{2}$ sensing with waveguides [2,3] required complex fabrication and yielded cladding power ratios below $10 \%$.

Here, we present a partially suspended mid-IR silicon (Si) photonic waveguide with low field confinement, and demonstrate the sensing of $\mathrm{CO}_{2}$ concentrations down to $0.1 \%$ with a $3.2 \mathrm{~mm}$ waveguide length. Our photonic circuit, manufacturable with a single lithography step, enables fully on-chip self-referenced gas measurements, to avoid characterization errors due to the $\mathrm{CO}_{2}$ present in the ambient air.
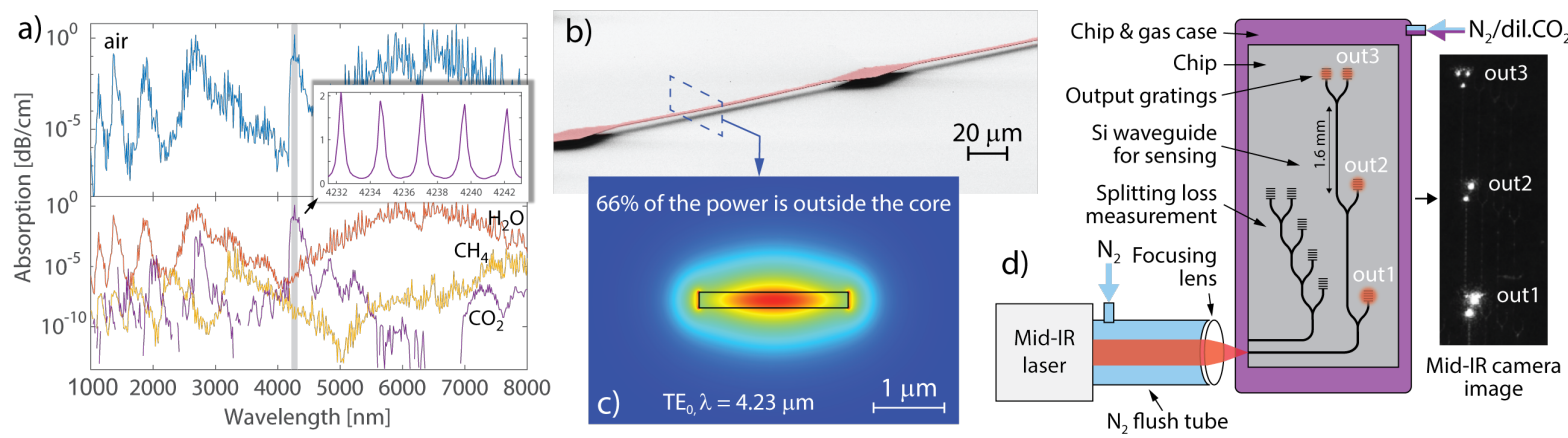

Fig. 1. a) Absorption spectra of air and its mid-IR-active constituents: water vapor, methane, and carbon dioxide [4]. b) SEM picture of our Si waveguide. c) Cross-sectional FEM simulation of the waveguide. d) Schematic of the measurement setup used for $\mathrm{CO}_{2}$ sensing and sample mid-IR image.

Our waveguide (Fig. 1b) is a partially suspended $\mathrm{Si}$ beam, supported by $\mathrm{SiO}_{2}$ pillars, at a separation of $3 \mu \mathrm{m}$ from the $\mathrm{Si}$ substrate. The single-TE-mode waveguide is $220 \mathrm{~nm}$ thick and $2.1 \mu \mathrm{m}$ wide in its suspended sections. Its simulated optical power fraction outside the core, and hence in the air cladding, is $66 \%$ (Fig. 1c). The waveguide layout (Fig. 1d) is designed for fully on-chip-referenced measurements. After an edge-coupled input section, three outputs branch out with symmetrical multi-mode interference splitters at regular length intervals of $1.6 \mathrm{~mm}$, and terminate in surface grating couplers. The waveguide was fabricated on an SOI substrate with a $220 \mathrm{~nm}$ Si device layer and a $3 \mu \mathrm{m} \mathrm{SiO} \mathrm{S}_{2} \mathrm{BOX}$ layer by a single e-beam lithography step, dry etching, wet etching, and cleaving.

We characterized the waveguide as a $\mathrm{CO}_{2}$ sensor with the setup shown in Fig. 1d. We coupled mid-IR light from a tunable quantum cascade laser into the waveguide with a focusing lens. The waveguide chip was placed 
inside a closed steel case with a mid-IR-transparent window that enabled the measurement of the intensity of the grating outputs with a mid-IR camera. We injected alternately nitrogen $\left(\mathrm{N}_{2}\right), 0.1 \%, 0.5 \%, 1 \%$, and $2.5 \% \mathrm{CO}_{2}$ diluted in $\mathrm{N}_{2}$, and again $\mathrm{N}_{2}$ at $600 \mathrm{~mL} \mathrm{~min}^{-1}$ into the case at intervals of $1 \mathrm{~min}$. The free-space light path sections between the laser head and the focusing lens and between the chip case window and the camera were flushed with $\mathrm{N}_{2}$. We continuously measured the waveguide propagation loss (Fig. 2a-d, bottom panels) during gas injection using the three output signals from the gratings (Fig. 2a-d, top panels) for a real-time cut-back measurement and subtracting the splitting loss previously measured in a separate experiment (Fig. 1d). For each $\mathrm{CO}_{2}$ concentration, we repeated the measurement at several different wavelengths, i.e. at different positions in $\mathrm{CO}_{2}$ absorption peak (Fig. 2f). The difference between the time-averaged waveguide propagation loss during $\mathrm{N}_{2}$ injection and the one during $\mathrm{CO}_{2}$ injection is the waveguide excess loss exclusively due to $\mathrm{CO}_{2}$ (Fig. 2e-f).
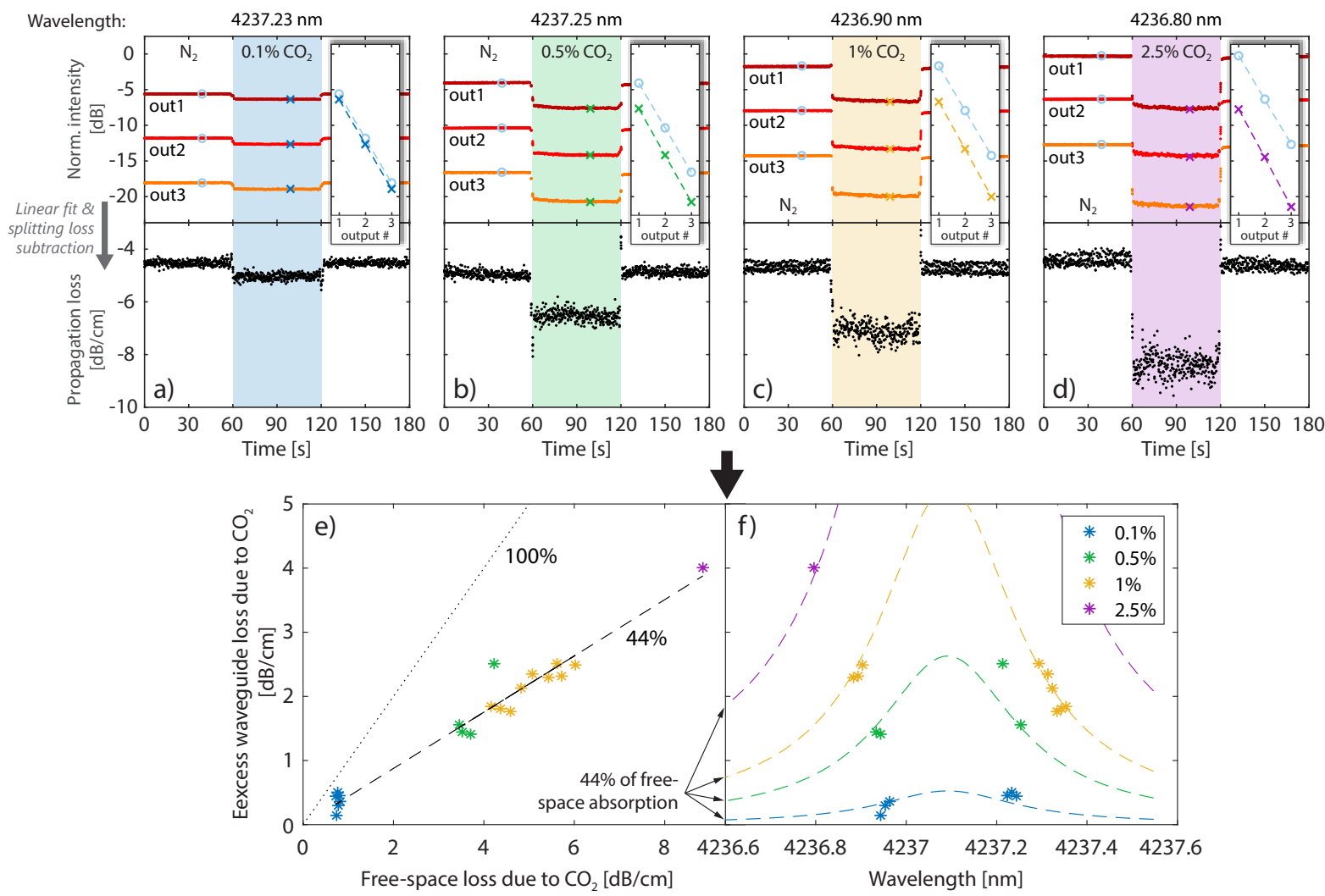

Fig. 2. a)-d) Four sample measurements of $0.1 \%, 0.5 \%, 1 \%$, and $2.5 \% \mathrm{CO}_{2}$ in $\mathrm{N}_{2}$, including the waveguide output signals and the extracted propagation loss. e) Measured excess waveguide loss due to $\mathrm{CO}_{2}$ compared to the free-space $\mathrm{CO}_{2}$ loss. f) Waveguide loss spectrum compared to $44 \%$ of the free-space loss spectrum as listed in the HITRAN database [4].

The waveguide has a base propagation loss of $4.6 \mathrm{~dB} / \mathrm{cm}$, and the effect of the $\mathrm{CO}_{2}$ injection on the propagation loss is clearly visible for all four $\mathrm{CO}_{2}$ concentrations. Fig. $2 \mathrm{~b}$ summarizes the measured excess loss due exclusively to the $\mathrm{CO}_{2}$ absorption along the waveguide, and relates it to the free-space $\mathrm{CO}_{2}$ absorption loss, as listed in the HITRAN database [4]. We found that the sensitivity of our waveguide to $\mathrm{CO}_{2}$ is $44 \%$ of the free-space sensitivity (Fig. 2e), the highest ever reported to our knowledge. The performance of our on-chip waveguide $\mathrm{CO}_{2}$ sensor, and its simple and cost-effective fabrication, make it ideal for mass production and commercialization for both existing and new applications, such as portable and distributed environmental monitoring, medical, and highvolume consumer applications.

\section{References}

1. J. Hodgkinson and R.P. Tatam, ”Optical Gas Sensing: A Review”, Meas. Sci. Technol., 24(1), 012004 (2013).

2. R. Siebert and J. Müller, "Infrared Integrated Optical Evanescent Field Sensor for Gas Analysis", Sens. Actuat. A, 119(2), 584-592 (2005).

3. C. Ranacher, C. Consani, N Vollert, A. Tortschanoff, M. Bergmeister, T. Grille, and B. Jakoby, "Characterization of Evanescent Field Gas Sensor Structures Based on Silicon Photonics", IEEE Photonics J., 10(5), 1-14 (2018).

4. I.E. Gordon, L.S. Rothman, C. Hill et al., "The HITRAN2016 Molecular Spectroscopic Database”, J. Quant. Spectrosc. Radiat. Transfer 203, 3-69 (2017). 